\title{
Spectrum of Skeletal Tuberculosis in Children
}

\author{
Pinheiro Pedro Carlos* \\ Orthopedic Department, Jesus Children`s Hospital, Rio de Janeiro, Brazil
}

Received: March 29, 2014; Accepted: May 13, 2014; Published: May 19, 2014

*Corresponding author: Pinheiro Pedro Carlos M. S, MD, PhD, Orthopedic Department, Jesus Children's Hospital, Rio de Janeiro, Brazil. CEP: 22.040-050; Tel: 55.021 2255-4685; E-mail: pecacho@infolink.com.br

\begin{abstract}
Treatment of tuberculosis remains controversial to date as it comprises a serious public health problem issue. Over the last 50 years, many empirical therapeutic compositions have been made. Throughout the world, immunodeficiency virus infection entails one of the diseases responsible for the increase in the number of cases in children. Its eradication has not been effected. Some works on spine tuberculosis (involving from $25 \%$ to $60 \%$ of cases) in children, support the conservative method, while others prefer surgical procedures. The purpose of this study is to investigate treatment results in children displaying skeletal osteoarticular tuberculosis manifestations, using the modern multidrug chemotherapy treatment protocol and cast immobilization.
\end{abstract}

Methods: The research was performed based on a study of the records of a conservative series of 94 patients diagnosed with osteoarticular tuberculosis. The sample enclosed in the study was that of children with pure bone tuberculosis or associated with other tuberculosis foci. All the patients were treated by our department's standard protocol, based on antituberculous drugs (rifampicim, isoniazid, pyrazinamide and ethambutol) plus cast immobilization. Statistical analysis was performed by means of a "one-way" study of variance and Kruskal-Wallis (nonparametric test). Tukey`s test was also used as well as the chi-square $\mathrm{X}^{2}$ or the Fisher accuracy test at a $5 \%$ level.

Results: A satisfactory result was obtained with conservative treatment (antituberculous drugs and cast immobilization) using our department's standard protocol. Significant relationship exists between the affected site and the age $(\mathrm{p}=0.012)$. The hip group showed a significant higher average age than the affected thoracic and lumbar spine group. A Significant relationship was observed between the affected hip and time in cast $(\mathrm{p}=0.0001)$. This means that the hip group presented a median time in cast significantly lower than the affected thoracic and lumbar spine group. There is no significant difference in follow-up time $(\mathrm{p}=0.54)$ between the hip, the thoracic and the lumbar spine.

Conclusions: After analyzing the therapy method based on antituberculous drugs and plaster cast immobilization, we conclude that employment of the standard protocol treatment, together with modern multidrug chemotherapy and cast were operational, justifiable and productive; it can also be used as an alternative therapeutic procedure for this specific disease.

Keywords: Tuberculosis; Bone tuberculosis; Cast immobilization

Level of evidence: Level - I Therapeutic Studies-Investigating the results of treatment Prognostic Studies.

\section{Introduction}

Before the advent of antibiotics, tuberculosis was a common and lethal disease, its incidence having decreased since the 1950s $[1,2]$. In the early 1980s, there was an increase in the number of cases, primarily attributed to AIDS (acquired immunodeficiency syndrome) and socioeconomic status deterioration $[2,3]$.

Extrapulmonary sites, such as the musculo-skeletal system, entail one of the most common forms in children, involvement occurring especially in the spine, hips and knee joints respectively [1,4]. Improving the population's living conditions is of utmost importance for social control of the disease. Children are vulnerable and more likely to have a serious, lethal risk of sequelae (osseous ankylosis) [4]. Early osteoarticular tuberculosis diagnosis is basic to the prevention of skeletal deformities [2,4-6]. The purpose of this study is to investigate treatment results in children displaying skeletal osteoarticular tuberculosis manifestations, using the modern multidrug chemotherapy treatment protocol and cast immobilization.

\section{History}

The history of tuberculosis is intermingled with the humanity history. Evidences of the disease were found in Egyptian mummy bones dating from $3400 \mathrm{BC}$ from archaeological excavations [6,7].

Hindu writings described the disease meaning "consumption" (or destruction by means of body deterioration; by progression of body condition). Hippocrates (460-337 BC) introduced the term for tuberculosis phthisis in ancient Greek, which has several meanings including: "to consume", "to spit" and "to waste away" [7].

Percival Pott, an Enghish surgeon, wrote, in his monograph, in 1779, the first description of spine tuberculosis. This author associated a painful marked spine kyphotic deformity with paraplegia. This condition came to be known as Pott`s disease. (spondylitis associated with this infection)[8].

Johan Schoenlein (1793-1864) introduced, in 1839, the word tuberculosis derived from the Latin word tubercula (the diminutive of tuber), which means a small lump [7].

It was only possible to identify the organism responsible for the disease in the second half of the nineteenth century. 
Mycobacterium tuberculosis was isolated in 1882 by Robert Koch a German Physician, Bacteriologist and Researcher 1843-1910) [7], who identified the Koch tubercle and reported his discovery to the Berlin Physiological Society, his paper being published in April 1882 [7].

\section{Background}

The contributions and reasons for using conservative multidrug chemotherapy and plaster cast treatment in the management of children with tuberculosis are as follows:

- consequence: early diagnosis and opportune treatment are basic to skeletal deformity prevention in children;

- explanation: conservative protocol treatment used showed that, with spine cases, surgical indication should be done in patients with no symptom regression, according to criteria in the literature;

- understanding: this work provides opportunity for orthopedic surgeons to employ a biology-based method, thereby fostering the possibility of wide debate on the subject;

- therapy attendance: utilization of the modern chemotherapeutic regime developed has proven its effectiveness in the treatment of skeletal tuberculosis in children, based on statistical data secured, thereby demonstrating its significance and suitability;

- course of the disease: the improvement of the patient's living conditions and the physician's and close follow-up favors good prognosis for the disease.

This study postulates that the advantage and applicability of the modern multidrug chemotherapy and cast immobilization can be included as a valid treatment method, considering the dubiousness of the course of any disease.

\section{Methods}

The local Ethics in Human Research Committee of the Municipal Secretariat for Health and Civil Defense of Rio de Janeiro (Brazil), under protocol no. 172/11, CAAE no. 0070.0.314.00011, approved the Research Project entitled "Skeletal tuberculosis in children," no external funding being received. Informed consent from the patient's parents /guardians for publication of this report and accompanying images has been obtained.

Typology of the design employed in this sample was a study of a single cohort with observational, intervention, longitudinal (follow-up) and retrospective characteristics. Antituberculous therapy and plaster cast were the dependent variables in this research. A consecutive series of 94 children affected by osteoarticular tuberculosis treated at the Jesus Children`s Hospital (Orthopedics and Traumatology Department) in the period of twenty years were managed by antituberculous drugs and the use of cast immobilization. Patients' ages varied at the time of diagnosis, ranging from 6 months, to age 11. The followup duration ranged from six months to 19 years, an average of 27.6 months. Forty-seven patients were males, and 47 females.
Regarding race, 20 were white and 74 non-white. According to the onset of symptoms, the disease was detected in 61 children in the first month; 17 in the second and, 16 patients were involved in the third. Pursuant to the Mantoux tuberculin skin test, 69 were performed and 25 were not-performed, 36 were strong reactors, 29 were non-reactors and 4 were weak reactors. Seventy-five cases $(80 \%)$ were found regarding spine level, 35 (37\%) being at the lumbar segment, $20(22 \%)$ thoracic, $4(4 \%)$ thoracolumbar, $3(3 \%)$ lumbar-sacrum, 2 (2\%) cervical, and $11(12 \%)$ without a specific affected spine segment. According to other bone location segments, there were $15(16 \%)$, hip patients, $1(1 \%)$ being femur, $1(1 \%)$ radius, $1(1 \%)$ hand, and $1(1 \%)$ affected in the sternum bone. AIDS serology tests were performed in nine patients, only one case being positive and eight negative. The treatment methods used were based on symptomatology; epidemiological clinical history, clinical examination, laboratory erythrocyte sedimentation rate (ESR), neurologic deficits, roentgenographic appearance. Bone biopsy in some patients was evaluated to confirm the diagnosis. Multidrug chemotherapy (nine months) and cast immobilization treatment regime was performed. A nine-month chemotherapy period was used as routine. Cast immobilization was carried out at a six-month average according to joint affected so as to produce pain, muscle spasms and local tenderness relief.

The following statistical analysis methods with the following objectives were performed:

a. In order to compare quantitative Data from three affected sites, analysis of one-way variance or Kruskal-Wallis (nonparametric ANOVA) was used. Tukey`s test and multiple comparison test was based on Kruskal-Wallis test statistical analysis were used to identify different sites; the chi-square $\mathrm{X}^{2}$ or Fisher accuracy test was applied to compare proportions (qualitative data).

b. Nonparametric tests were carried out at because time in cast and follow-up time, did not present normal distribution (Gaussian), due to wide dispersion and lack of symmetry of the distribution.

c. The determining significance criterion was set at $5 \%$. i.e., when the $\mathrm{p}$ value $\leq 0.005$, then there is statistical significance. Statistical analysis using the statistical software SAS $®$ System 6.04 was utilized.

\section{Treatment Protocol}

The main objective of skeletal tuberculosis treatment is the need for the earliest, the safest and the most effective diagnosis to avoid disease progression, reducing illness duration, improving nutrition, providing weight gain, relieving the pain and terminating unexplained fever.

The adequate therapy method was based on the combination of a modern standard protocol using four antituberculous drugs. The protocol used at our department for 9 months involved four drugs in two phases: firstly, the intensive phase: Rifampicin 10 $\mathrm{mg} / \mathrm{kg} /$ day, (2 months) Isoniazid (isonicotinic acid hydrazide) INH: $10 \mathrm{mg} / \mathrm{kg} /$ day, (2 months), and Pyrazinamide 35mg/kg/ 
day, (2 months). Secondly, the maintenance phase: Rifampicim $10 \mathrm{mg} / \mathrm{kg} /$ day, (7 months) and Isoniazid (isonicotinic acid hydrazide) INH: $10 \mathrm{mg} / \mathrm{kg} /$ day (7 months). Ethambutol 25mg/ $\mathrm{kg} /$ day was included in the first intensive phase for patients over 10 years old. The criteria adopted for protocol interruption after 9 months were based on the patients' favorable condition and symptoms, when those symptoms have decreased. The disease can be considered successfully treated when the patient's health is restored, their abscesses disappear, the X-rays are radiologically inactive, fever is absent, normal hematological testing white blood cell count laboratory rates are achieved, and the patient is free of systemic complaints.

\section{Results}

The factors used in the descriptive statistical analysis of our results study were as follows: a) disease location, b) orthopedic treatment performed and immobilization time. The previouslymentioned multidrug therapy protocol for tuberculosis was applied to all patients. An average of 4.7 osteoarticular tuberculosis cases per year was processed at our department.

The period between the onset of symptoms, diagnosis and treatment of the disease was less than a month in 31 cases (33\%), more than a month $30(31 \%)$, more than two in 17 (18\%) and more than three in 16 (17\%).

Tuberculosis was developed in $32 \%$ of cases, which had a Bacille Calmette-Guérin (BCG) vaccination scar, confirming that the vaccine safely protects only against severe forms, such as miliar and meningitis tuberculosis.

The average follow-up period was 10 months due to the irregularity in attendance patient to the clinic on account of symptom by improvement shortly after starting chemotherapy treatment with the regime established. In addition, annual distribution showed a declining trend in the last five years. Pursuant to the data, there was no relevance in the distribution of cases and time of the year.

The most frequent symptoms observed were: pain, local functional limitation, and swelling. In cases of spondylitis tuberculosis, severe kyphosis with abscesses paraparesis/ paraplegia could be detected. An improvement of pain and neurological examinations after initiation of modern chemotherapy protocol treatment was obtained within two months.

One patient had concomitant involvement of lumbar spine and the knee joint (Case 20) and another case thoracic spine with the elbow joint (Case 60) was found.

A Minerva plaster cast jacket type and a protective cervical collar were used in two patients (2\% [Cases 9 and 89]) with cervical spine compromising. In cases with the thoracic or lumbar spine involvement, plaster jackets, including both hips and thighs, were applied in 55 (59\%) of the children and 17 $(18 \%)$ thoracolumbar plaster jacket. Spica casts were used in 15 patients $(16 \%)$ with hip osteoarticular tuberculosis. A cast with metacarpal heads to the axilla was applied in elbow joint tuberculosis (Case 60).
Spica and metacarpal heads-to-the-axilla casts respectively were used in patients with femur and radius osteomyelites tuberculosis (Cases 80 and 94). Children with sternum and arthritis tuberculosis of the hand associated with dactylitis, (Cases 82 and 93) respectively, were not subjected to cast immobilization just as patients with spine and knee joint tuberculosis (Case 20).

The average cast immobilization length was 4.7 months. Patients who used plaster jackets including both hips and thighs were on average immobilized for five months. Two patients (Cases 9 and 89) with cervical spondylitis tuberculosis remained on average 42 days with a Minerva plaster cast jacket type. After that, a cervical collar was placed for 4 months.

Three patients (3.1\% [Cases 57, 75, and 91]) with spine and iliopsoas abscesses involvement underwent surgical drainage and spica cast immobilization two other cases (2.1\% [Cases 49 and 92]) with spine involvement showed lumbar abscesses; in which surgical drainage and plastered immobilization were performed. Spinal fusion was performed in four patients $(4.2 \%$ [Cases 19, 20, 26, and 31]) in an effort to avoid progression of spine deformation (kyphosis) and neurological complications. The Albee [9] and Hibbs [10] technique with autologous bone graft from the tibia (Cases 23 and 24) was used.

Two patients (2.1\% [Cases 1 and 7]) with hip involvement later underwent femoral subtrochanteric valgus osteotomy in an attempt to improve neck femoral shaft angle relationship. Five other patients (5.3\% [Cases 34, 46, 50, 69, and 76]) were submitted to hip joint puncture biopsy, followed by arthrotomy, and spica cast immobilization.

Patients with osteomyelites tuberculosis of femur, sternum, and radius (Cases 80,93 , and 94) were submitted to decompressive needle puncture biopsy for diagnostic purposes.

Two patients (Cases 95 and 96) with cervical segment tuberculosis were excluded from the study. Both patients had lymph node tuberculosis in the neck, denominated scrofula, a form of tuberculosis connecting the head and the trunk (neck).

\section{Statistical Analysis}

\section{First statistical objective}

General profile of the casuistry, follow-up time of 94 patients, average standard deviation (SD), median, minimum and maximum age and time in cast were studied (Table 1).

\section{Second statistical objective}

To specify whether there was significant association between the local region affected with clinical and demographic variables. This analysis aimed to observe whether the three groups (hip, thoracic and lumbar spine) were statistically different according to clinical and demographic variables in association with the qualitative variables. The table below provides the frequency (n) and percentage (\%) of the qualitative variables according to the affected site and the descriptive correspondence level ( $\mathrm{p}$ value) of the statistical test. Statistical analysis by $\mathrm{X}^{2}$ or by Fisher (qualitative data) exact test was held (Table 2). 


\section{Third statistical objective}

The table below provides the average, standard deviation (SD), median, minimum and maximum age, length of follow-up plaster in months, the second involvement sites, and the statistical test corresponding descriptive level ( $p$ value). Statistical analysis by analysis of variance (age) and Kruskal-Wallis ANOVA (time in cast and follow-up) were performed. The different sites were identified by multiple comparison test corresponding to the $5 \%$ level described in the "significant differences" column (Table 3,4).

\section{Discussion}

There is some disagreement over the sex prevalence of the disease. The literature usually shows male predominance
[3,5,6,11-15] over the females. No gender preference was observed in this study (50\%).

Regarding race types, a higher incidence among non-whites $(80 \%)$ rather than white $(20 \%)$ patients was observed. This was due to the Brazilian population's interracial mix.

The Mantoux test is a routine medical exam used in pediatrics to help in the tuberculosis infection diagnosis. It was expected all children in the sample to be reactive to Mantoux test because vaccination with BCG (Bacille Calmette-Guérin) is a routine procedure immunization in Brazil. In this work, $42 \%$ of the cases submitted to the Mantoux test displayed no reaction in the patients. There are several explanations for these factors related

Table 1: Descriptive numerical characteristics analysis.

\begin{tabular}{|c|c|c|c|c|c|}
\hline Variable & n & Average & SD & Median & Minimum \\
\hline Age (months) & 94 & 42,2 & 28,4 & 34 & 132 \\
\hline Time in Cast (days) & 92 & 141,3 & 48,5 & 122,5 & 15 \\
\hline Follow-up (months) & 94 & 27,7 & 36,8 & 10 & 4,6 \\
\hline
\end{tabular}

$\mathrm{n}=$ number of cases; $\mathrm{SD}=$ standard deviation

Table 2: Demographic variables and qualitative clinics according to affected site.

\begin{tabular}{|c|c|c|c|c|c|c|c|c|}
\hline \multirow[b]{2}{*}{ Variable } & \multirow[b]{2}{*}{ Category } & \multicolumn{2}{|c|}{ Hip } & \multicolumn{2}{|c|}{ Thoracic Spine } & \multicolumn{2}{|c|}{$\begin{array}{c}\text { Lumbar } \\
\text { Spine }\end{array}$} & \multirow[b]{2}{*}{ p value } \\
\hline & & $\mathbf{n}$ & $\%$ & $\mathbf{n}$ & $\%$ & $\mathbf{n}$ & $\%$ & \\
\hline \multirow[b]{2}{*}{ Sex } & Male & 6 & 37.5 & 11 & 55.0 & 16 & 47.1 & \multirow{2}{*}{0.57} \\
\hline & Female & 10 & 62.5 & 9 & 45.0 & 18 & 52.9 & \\
\hline \multirow[b]{2}{*}{ Race } & White & 1 & 6.2 & 3 & 15.0 & 8 & 23.5 & \multirow{2}{*}{0.31} \\
\hline & Nonwhite & 15 & 93.8 & 17 & 85.0 & 26 & 76.5 & \\
\hline Mantoux test & Reactor & 10 & 71.4 & 14 & 82.4 & 8 & 34.8 & 0.006 \\
\hline \multirow{2}{*}{ Onset of symptoms } & $1^{\circ}$ month & 9 & 56.2 & 15 & 75.0 & 25 & 73.5 & \multirow{2}{*}{0.39} \\
\hline & $2^{\circ} / 3^{\circ}$ months & 7 & 43.8 & 5 & 25.0 & 9 & 26.5 & \\
\hline
\end{tabular}

It was observed that the patients' group with affected lumbar spine, in reaction with the Mantoux, test presented no reaction (65.2\%) significantly higher $(\mathrm{p}=0.006)$ than the affected hip patients' group (28.6\%) and the thoracic spine group (17.7\%). Significant difference in sex ( $\mathrm{p}=0.57)$, in race $(p=0.31)$ and in the onset of symptoms $(p=0.39)$ were observed among the three segments (hip, thoracic spine and lumbar spine) under study.

Table 3: Clinical and demographic variables, according to the affected local site.

\begin{tabular}{|c|c|c|c|c|c|c|c|c|c|}
\hline Varible & $\begin{array}{l}\text { Affected } \\
\text { Site }\end{array}$ & $\mathbf{n}$ & Average & SD & Median & Minimum & Maximum & $\begin{array}{c}\text { p } \\
\text { value }\end{array}$ & $\begin{array}{l}\text { significant } \\
\text { differences }\end{array}$ \\
\hline \multirow{3}{*}{ Age(Months) } & Hip & 16 & 55,4 & 25,3 & 55,5 & 6 & 108 & \multirow{3}{*}{0,012} & Hip $\neq$ Thoracic Spine \\
\hline & Thoracic Spine & 20 & 31,3 & 12,2 & 32 & 13 & 58 & & Hip $\neq$ Lumbar Spine \\
\hline & Lumbar Spine & 34 & 37,5 & 28,8 & 26 & 12 & 130 & & \\
\hline \multirow{2}{*}{$\begin{array}{c}\text { Time in Cast } \\
\text { (Days) }\end{array}$} & Hip & 16 & 98,8 & 33,0 & 90 & 30 & 160 & \multirow{2}{*}{0,0001} & Hip $\neq$ Thoracic Spine \\
\hline & Thoracic Spine & 20 & 169,5 & 38,6 & 180 & 105 & 270 & & Hip $\neq$ Lumbar Spine \\
\hline \multirow{3}{*}{$\begin{array}{l}\text { Follow-up } \\
\text { (Months) }\end{array}$} & Hip & 16 & 35,8 & 56,6 & 12,55 & 4,6 & 230 & \multirow{3}{*}{0,54} & \\
\hline & Thoracic Spine & 20 & 31,3 & 30,9 & 15 & 6 & 93,3 & & \\
\hline & Lumbar Spine & 34 & 25,6 & 35,7 & 9 & 6 & 146,6 & & \\
\hline
\end{tabular}

$\mathrm{Sd}=$ Standard Deviation; $\mathrm{N}=$ Number Of Cases.

The following was observed a: there is a significant relationship between the affected region and the patient`s age $(p=0.012)$. this means that the affected hip group showed a significantly higher average age than the group with the affected thoracic and lumbar spine. b: there is significant relationship between the affected site and time in cast $(p=0.0001)$. this means that the affected hip group showed a median time in cast significantly lower than the group with the affected thoracic and lumbar spine group. $c$ : there is no significant difference in follow-up time ( $\mathrm{p}=0.54$ ) among the hip, the thoracic and the lumbar spine groups. 
Table 4: Data on the Patients.

\begin{tabular}{|c|c|c|c|c|c|c|c|c|c|c|c|}
\hline Case & $\begin{array}{l}\text { Age At } \\
\text { (Yrs } \\
\text { Month) }\end{array}$ & Sex* & Race $^{\#}$ & BCG & PPD & $\begin{array}{c}\text { Onset of } \\
\text { Symptoms }\end{array}$ & Affected Area & Type of Cast & $\begin{array}{l}\text { Time In } \\
\text { Cast } \\
\text { (Days) }\end{array}$ & Surgical Treatment & $\begin{array}{l}\text { Follow- } \\
\text { Up } \\
\text { (Months }\end{array}$ \\
\hline 1 & $5 / 9$ & $\mathrm{f}$ & $\mathrm{N}-\mathrm{W}$ & - & $\begin{array}{c}\text { No } \\
\text { Reactor }\end{array}$ & +3 Months & Left Hip & Spica Cast & 150 & $\begin{array}{c}\text { Valgus Subtrocanteric } \\
\text { Femoral Osteotomy }\end{array}$ & 36.5 \\
\hline 2 & $4 / 5$ & $\mathrm{~F}$ & $\mathrm{~N}-\mathrm{w}$ & Yes & $\begin{array}{l}\text { Strong } \\
\text { Reactor }\end{array}$ & - 1 Month & Right Hip & Spica Cast & 90 & - & 13.1 \\
\hline 3 & $5 / 11$ & $\mathrm{~F}$ & $\mathrm{~N}-\mathrm{w}$ & - & $\begin{array}{c}\text { No- } \\
\text { Reactor }\end{array}$ & + 1 Month & Right Hip & Spica Cast & 100 & - & 12 \\
\hline 4 & $3 / 8$ & $\mathrm{~m}$ & w & Yes & $\begin{array}{l}\text { No- } \\
\text { Reactor }\end{array}$ & + 1 Month & Spine & $\begin{array}{l}\text { Plaster Jackets } \\
\text { including both } \\
\text { hips and thighs }\end{array}$ & 180 & - & 13.1 \\
\hline 5 & $2 / 0$ & $\mathrm{~F}$ & $\mathrm{~N}-\mathrm{w}$ & - & - & + 3 Months & Spine & $\begin{array}{l}\text { Plaster Jackets } \\
\text { including both } \\
\text { hips and thighs }\end{array}$ & 180 & - & 19.6 \\
\hline 6 & $2 / 3$ & $\mathrm{~m}$ & w & - & $\begin{array}{l}\text { Strong } \\
\text { Reactor }\end{array}$ & + 1 Month & $\begin{array}{c}\text { Lumbar Spine } \\
\text { L1-L2 }\end{array}$ & $\begin{array}{l}\text { Plaster Jackets } \\
\text { including both } \\
\text { hips and thighs }\end{array}$ & 100 & - & 8 \\
\hline 7 & $3 / 8$ & $\mathrm{~F}$ & $\mathrm{~N}-\mathrm{w}$ & - & $\begin{array}{l}\text { Strong } \\
\text { Reactor }\end{array}$ & +1 Month & Right Hip & Spica Cast & 90 & $\begin{array}{l}\text { Punch/Artrotomy/Biopsy } \\
\text { Valgus Subtrocanteric } \\
\text { Femoral Osteotomy }\end{array}$ & 66.6 \\
\hline 8 & $2 / 3$ & $\mathrm{~m}$ & $\mathrm{~N}-\mathrm{w}$ & No & $\begin{array}{l}\text { Strong } \\
\text { Reactor }\end{array}$ & + 2 Months & $\begin{array}{c}\text { Thoracic Spine } \\
\text { T8-T9 }\end{array}$ & $\begin{array}{c}\text { Thoracolumbar } \\
\text { Cast }\end{array}$ & 180 & - & 8.3 \\
\hline 9 & $2 / 7$ & $\mathrm{~m}$ & $\mathrm{~N}-\mathrm{w}$ & - & $\begin{array}{l}\text { Strong } \\
\text { Reactor }\end{array}$ & + 3 Months & $\begin{array}{c}\text { Cervical Spine } \\
\text { C5-C6 }\end{array}$ & $\begin{array}{l}\text { Minerva Jacket } \\
\text { Cervical Collar }\end{array}$ & $\begin{array}{l}45 \\
45\end{array}$ & - & 6 \\
\hline 10 & $1 / 5$ & $\mathrm{~F}$ & $\mathrm{~N}-\mathrm{w}$ & - & - & +2 Months & $\begin{array}{c}\text { Lumbar Spine } \\
\text { L1-L2 }\end{array}$ & $\begin{array}{l}\text { Plaster Jackets } \\
\text { including both } \\
\text { hips and thighs }\end{array}$ & 180 & - & 7 \\
\hline 11 & $2 / 4$ & $\mathrm{~F}$ & $\mathrm{w}$ & - & - & + 1 Month & $\begin{array}{c}\text { Lumbar Spine } \\
\text { L3-L4 }\end{array}$ & $\begin{array}{l}\text { Plaster Jackets } \\
\text { including both } \\
\text { hips and thighs }\end{array}$ & 180 & - & 10 \\
\hline 12 & $4 / 10$ & $\mathrm{~F}$ & $\mathrm{~N}-\mathrm{w}$ & - & $\begin{array}{l}\text { Strong } \\
\text { Reactor }\end{array}$ & +2 Months & Left Hip & Spica Cast & 160 & - & 10 \\
\hline 13 & $1 / 8$ & $\mathrm{f}$ & $\mathrm{w}$ & - & $\begin{array}{c}\text { No- } \\
\text { Reactor }\end{array}$ & +2 Months & $\begin{array}{c}\text { Lumbar Spine } \\
\text { L4-L5 }\end{array}$ & $\begin{array}{l}\text { Plaster Jackets } \\
\text { including both } \\
\text { hips and thighs }\end{array}$ & 180 & - & 60 \\
\hline 14 & $1 / 4$ & $\mathrm{~m}$ & $\mathrm{~N}-\mathrm{w}$ & - & $\begin{array}{l}\text { Stong } \\
\text { Reactor }\end{array}$ & - 1 Month & $\begin{array}{c}\text { Thoracic Spine } \\
\text { T7-T8-T9 }\end{array}$ & $\begin{array}{l}\text { Plaster Jackets } \\
\text { including both } \\
\text { hips and thighs }\end{array}$ & 180 & - & 76.6 \\
\hline 15 & $3 / 0$ & $\mathrm{~m}$ & $\mathrm{~N}-\mathrm{w}$ & - & $\begin{array}{c}\text { Weak } \\
\text { Reactor }\end{array}$ & - 1 Month & $\begin{array}{c}\text { Thoracic Spine } \\
\text { L T11-T12 }\end{array}$ & $\begin{array}{l}\text { Plaster Jackets } \\
\text { including both } \\
\text { hips and thighs }\end{array}$ & 120 & - & 20 \\
\hline 16 & $3 / 0$ & $\mathrm{~m}$ & w & Yes & $\begin{array}{c}\text { No- } \\
\text { Reactor }\end{array}$ & - 1 Month & $\begin{array}{l}\text { Thoracolumbar } \\
\text { Spine T12-L1 }\end{array}$ & $\begin{array}{l}\text { Plaster Jackets } \\
\text { including both } \\
\text { hips and thighs }\end{array}$ & 120 & - & 6 \\
\hline 17 & $2 / 5$ & $\mathrm{~m}$ & w & Yes & - & - 1 Month & Spine & $\begin{array}{l}\text { Plaster Jackets } \\
\text { including both } \\
\text { hips and thighs }\end{array}$ & 160 & - & 6.6 \\
\hline 18 & $2 / 1$ & $\mathrm{~F}$ & $\mathrm{~N}-\mathrm{w}$ & Yes & - & + 1 Month & $\begin{array}{c}\text { Lumbar Spine } \\
\text { L1-L2 }\end{array}$ & $\begin{array}{l}\text { Plaster Jackets } \\
\text { including both } \\
\text { hips and thighs }\end{array}$ & 105 & - & 6 \\
\hline 19 & $1 / 8$ & $\mathrm{~F}$ & $\mathrm{w}$ & - & $\begin{array}{l}\text { Strong } \\
\text { Reactor }\end{array}$ & - 1 Month & $\begin{array}{c}\text { Thoracic Spine } \\
\text { T11-T12 }\end{array}$ & $\begin{array}{l}\text { Plaster Jackets } \\
\text { including both } \\
\text { hips and thighs }\end{array}$ & 170 & $\begin{array}{c}\text { Albee-Hibbs Vertebral } \\
\text { Arthrodesis }\end{array}$ & 24.3 \\
\hline 20 & $3 / 0$ & $\mathrm{~F}$ & N-W & Yes & - & +3 Months & $\begin{array}{c}\text { Lumbar Spine } \\
\text { L415/Right } \\
\text { Knee }\end{array}$ & $\begin{array}{l}\text { Plaster Jackets } \\
\text { including both } \\
\text { hips and thighs }\end{array}$ & 200 & $\begin{array}{c}\text { Albee-Hibbs Vertebral } \\
\text { Arthrodesis }\end{array}$ & 13.1 \\
\hline
\end{tabular}




\begin{tabular}{|c|c|c|c|c|c|c|c|c|c|c|c|}
\hline 21 & $1 / 6$ & M & $\mathrm{N}-\mathrm{W}$ & Yes & - & +2 Months & Spine & $\begin{array}{l}\text { Plaster Jackets } \\
\text { including both } \\
\text { hips and thighs }\end{array}$ & 180 & - & 6.6 \\
\hline 22 & $7 / 4$ & M & $\mathrm{N}-\mathrm{W}$ & - & $\begin{array}{c}\text { No } \\
\text { Reactor }\end{array}$ & + 1 Month & Spine & $\begin{array}{l}\text { Plaster Jackets } \\
\text { including both } \\
\text { hips and thighs }\end{array}$ & 100 & - & 6 \\
\hline 23 & $11 / 0$ & $\mathrm{~F}$ & W & - & $\begin{array}{l}\text { Strong } \\
\text { Reactor }\end{array}$ & - 1 Month & $\begin{array}{c}\text { Lumbar Spine } \\
\text { L5-S1 }\end{array}$ & $\begin{array}{l}\text { Plaster Jackets } \\
\text { including both } \\
\text { hips and thighs }\end{array}$ & 90 & - & 60 \\
\hline 24 & $3 / 0$ & M & $\mathrm{N}-\mathrm{W}$ & - & - & +2 Months & Spine T12-L1 & $\begin{array}{l}\text { Plaster Jackets } \\
\text { including both } \\
\text { hips and thighs }\end{array}$ & 90 & - & 6 \\
\hline 25 & $2 / 4$ & $\mathrm{~F}$ & $\mathrm{~N}-\mathrm{W}$ & - & - & - 1 Month & Spine & $\begin{array}{l}\text { Plaster Jackets } \\
\text { including both } \\
\text { hips and thighs }\end{array}$ & 100 & - & 6 \\
\hline 26 & $3 / 0$ & $\mathrm{~F}$ & $\mathrm{~N}-\mathrm{W}$ & - & - & - 1 Month & Spine T6-T9 & $\begin{array}{c}\text { Thoracolumbar } \\
\text { Cast }\end{array}$ & 270 & $\begin{array}{c}\text { Albee-Hibbs Vertebral } \\
\text { Arthrodesis }\end{array}$ & 70 \\
\hline 27 & $10 / 8$ & M & $\mathrm{N}-\mathrm{W}$ & - & - & - 1 Month & Spine & $\begin{array}{c}\text { Thoracolumbar } \\
\text { Cast }\end{array}$ & 120 & - & 8 \\
\hline 28 & $1 / 1$ & $\mathrm{~F}$ & $\mathrm{~N}-\mathrm{w}$ & - & $\begin{array}{c}\text { No- } \\
\text { Reactor }\end{array}$ & - 1 Month & Spine L4-L5 & $\begin{array}{l}\text { Plaster Jackets } \\
\text { including both } \\
\text { hips and thighs }\end{array}$ & 180 & - & 22.3 \\
\hline 29 & $4 / 1$ & $\mathrm{~F}$ & W & Yes & $\begin{array}{c}\text { No- } \\
\text { Reactor }\end{array}$ & - 1 Month & Spine L3-L4 & $\begin{array}{l}\text { Plaster Jackets } \\
\text { including both } \\
\text { hips and thighs }\end{array}$ & 120 & - & 19 \\
\hline 30 & $2 / 8$ & $\mathrm{~F}$ & $\mathrm{~W}$ & Yes & $\begin{array}{c}\text { No- } \\
\text { Reactor }\end{array}$ & -1 Month & Spine L4-L5 & $\begin{array}{l}\text { Plaster Jackets } \\
\text { including both } \\
\text { hips and thighs }\end{array}$ & 105 & - & 6 \\
\hline 31 & $4 / 10$ & M & $\mathrm{N}-\mathrm{W}$ & Yes & - & +3 Months & Spine $\mathrm{T} 10-\mathrm{T} 11$ & $\begin{array}{l}\text { Plaster Jackets } \\
\text { including both } \\
\text { hips and thighs }\end{array}$ & 180 & $\begin{array}{l}\text { Albee-Hibbs Vertebral } \\
\text { Arthrodesis }\end{array}$ & 93.3 \\
\hline 32 & $4 / 0$ & M & $\mathrm{N}-\mathrm{W}$ & - & $\begin{array}{c}\text { No- } \\
\text { Reactor }\end{array}$ & - 1 Month & Left Hip & Spica Cast & 90 & - & 230 \\
\hline 33 & $3 / 8$ & M & W & Yes & - & - 1 Month & Left Hip & Spica Cast & 120 & - & 6 \\
\hline 34 & $9 / 0$ & M & $\mathrm{N}-\mathrm{W}$ & - & - & +2 Months & Left Hip & Spica Cast & 90 & $\begin{array}{c}\text { Puncture/Artrotomy/ } \\
\text { Biopsy }\end{array}$ & 60 \\
\hline 35 & $6 / 0$ & $\mathrm{~F}$ & $\mathrm{~N}-\mathrm{W}$ & - & $\begin{array}{l}\text { Strong } \\
\text { Reator }\end{array}$ & - 1 Month & Left Hip & Spica Cast & 60 & - & 10 \\
\hline 36 & $2 / 1$ & $\mathrm{~F}$ & W & - & $\begin{array}{c}\text { No- } \\
\text { Reactor }\end{array}$ & - 1 Month & Spine L1-L2 & $\begin{array}{l}\text { Plaster Jackets } \\
\text { including both } \\
\text { hips and thighs }\end{array}$ & 180 & - & 26.6 \\
\hline 37 & $8 / 7$ & M & $\mathrm{W}$ & - & $\begin{array}{c}\text { No- } \\
\text { Reactor }\end{array}$ & - 1 Month & Spine L4-L5 & $\begin{array}{l}\text { Plaster Jackets } \\
\text { including both } \\
\text { hips and thighs }\end{array}$ & 180 & - & 143 \\
\hline 38 & $5 / 6$ & M & $\mathrm{N}-\mathrm{W}$ & - & - & +2 Months & Spine L3-L4 & $\begin{array}{c}\text { Thoracolumbar } \\
\text { Cast }\end{array}$ & 150 & - & 80 \\
\hline 39 & $3 / 2$ & M & $\mathrm{W}$ & - & $\begin{array}{l}\text { Strong } \\
\text { Reactor }\end{array}$ & +2 Months & Spine T7-T8-T9 & $\begin{array}{c}\text { Thoracolumbar } \\
\text { Cast }\end{array}$ & 125 & - & 6 \\
\hline 40 & $1 / 1$ & M & $\mathrm{W}$ & - & $\begin{array}{c}\text { No- } \\
\text { Reactor }\end{array}$ & - 1 Month & Spine L4-L5 & $\begin{array}{l}\text { Plaster Jackets } \\
\text { including both } \\
\text { hips and thighs }\end{array}$ & 120 & - & 23.3 \\
\hline 41 & $1 / 1$ & M & $\mathrm{N}-\mathrm{W}$ & - & $\begin{array}{c}\text { No- } \\
\text { Reactor }\end{array}$ & - 1 Month & Spine L3-L4 & $\begin{array}{l}\text { Plaster Jackets } \\
\text { including both } \\
\text { hips and thighs }\end{array}$ & 180 & - & 48. \\
\hline 42 & $1 / 4$ & M & $\mathrm{N}-\mathrm{W}$ & - & & - 1 Month & Spine L3-L4 & $\begin{array}{l}\text { Plaster Jackets } \\
\text { including both } \\
\text { hips and thighs }\end{array}$ & 110 & - & 9 \\
\hline 43 & $1 / 10$ & $\mathrm{~F}$ & $\mathrm{~N}-\mathrm{W}$ & - & - & +1 Month & Spine L3-L4 & $\begin{array}{l}\text { Plaster Jackets } \\
\text { including both } \\
\text { hips and thighs }\end{array}$ & 180 & - & 6.6 \\
\hline
\end{tabular}

Citation: Carlos PP (2014) Spectrum of Skeletal Tuberculosis in Children. J Exerc Sports Orthop 1(2): 1-10. DOI: http://dx.doi. Page 6 of 10 org/10.15226/2374-6904/1/2/00109 


\begin{tabular}{|c|c|c|c|c|c|c|c|c|c|c|c|}
\hline 44 & $2 / 6$ & $\mathrm{~F}$ & $\mathrm{~N}-\mathrm{W}$ & - & $\begin{array}{l}\text { Strong } \\
\text { Reactor }\end{array}$ & + 1 Month & Spine T3-T4 & $\begin{array}{c}\text { Thoracolumbar } \\
\text { Cast }\end{array}$ & 170 & - & 8 \\
\hline 45 & $1 / 6$ & M & $\mathrm{N}-\mathrm{W}$ & - & - & +2 Months & Spine T12-L1 & $\begin{array}{l}\text { Plaster Jackets } \\
\text { including both } \\
\text { hips and thighs }\end{array}$ & 120 & - & 83.3 \\
\hline 46 & $2 / 3$ & $\mathrm{~F}$ & $\mathrm{~N}-\mathrm{W}$ & - & $\begin{array}{c}\text { Weak } \\
\text { Reactor }\end{array}$ & +1 Month & Left Hip & Spica Gessada & 120 & $\begin{array}{c}\text { Puncture/Artrotomy/ } \\
\text { Biopsy }\end{array}$ & 14 \\
\hline 47 & $1 / 2$ & $\mathrm{~F}$ & $\mathrm{~N}-\mathrm{W}$ & - & $\begin{array}{c}\text { No- } \\
\text { Reactor }\end{array}$ & +1 Month & Spine L4-L5 & $\begin{array}{l}\text { Plaster Jackets } \\
\text { including both } \\
\text { hips and thighs }\end{array}$ & 180 & - & 19 \\
\hline 48 & $1 / 2$ & $\mathrm{~F}$ & $\mathrm{~N}-\mathrm{W}$ & - & $\begin{array}{c}\text { No- } \\
\text { Reactor }\end{array}$ & - 1 Month & Spine T9-T10 & $\begin{array}{c}\text { Thoracolumbar } \\
\text { Cast }\end{array}$ & 180 & - & 8 \\
\hline 49 & $3 / 5$ & $\mathrm{~F}$ & $\mathrm{~N}-\mathrm{W}$ & - & $\begin{array}{l}\text { Strong } \\
\text { Reactor }\end{array}$ & +1 Month & Spine T6-T7-T8 & $\begin{array}{c}\text { Thoracolumbar } \\
\text { Cast }\end{array}$ & 180 & Lumbar Abscess Drainage & 7.3 \\
\hline 50 & $6 / 11$ & M. & $\mathrm{N}-\mathrm{W}$ & - & $\begin{array}{l}\text { Strong } \\
\text { Reactor }\end{array}$ & + 1 Month & Right Hip & Spica Cast & 60 & $\begin{array}{c}\text { Puncture/Artrotomy/ } \\
\text { Biopsy }\end{array}$ & 70 \\
\hline 51 & $5 / 10$ & $\mathrm{M}$ & $\mathrm{N}-\mathrm{W}$ & - & $\begin{array}{c}\text { No- } \\
\text { Reactor }\end{array}$ & +1 Month & Spine L3-L4 & $\begin{array}{l}\text { Plaster Jackets } \\
\text { including both } \\
\text { hips and thighs }\end{array}$ & 90 & - & 8.3 \\
\hline 52 & $3 / 10$ & $\mathrm{M}$ & $\mathrm{N}-\mathrm{W}$ & - & - & + 1 Month & Spine L4-L5 & $\begin{array}{l}\text { Plaster Jackets } \\
\text { including both } \\
\text { hips and thighs }\end{array}$ & 90 & - & 8 \\
\hline 53 & $1 / 1$ & $\mathrm{~F}$ & W & Yes & $\begin{array}{c}\text { No- } \\
\text { Reactor }\end{array}$ & + 1 Month & Spine T11-T12 & $\begin{array}{l}\text { Plaster Jackets } \\
\text { including both } \\
\text { hips and thighs }\end{array}$ & 210 & - & 90 \\
\hline 54 & $7 / 6$ & M & $\mathrm{N}-\mathrm{W}$ & - & $\begin{array}{l}\text { Strong } \\
\text { Reactor }\end{array}$ & +2 Months & Spine & $\begin{array}{l}\text { Plaster Jackets } \\
\text { including both } \\
\text { hips and thighs }\end{array}$ & 180 & - & 12 \\
\hline 55 & $1 / 5$ & $\mathrm{~F}$ & $\mathrm{~N}-\mathrm{W}$ & Yes & $\begin{array}{c}\text { No- } \\
\text { Reator }\end{array}$ & + 2 Months & Spine L2-L3 & $\begin{array}{l}\text { Plaster Jackets } \\
\text { including both } \\
\text { hips and thighs }\end{array}$ & 150 & - & 6.6 \\
\hline 56 & $6 / 3$ & $\mathrm{M}$ & $\mathrm{N}-\mathrm{W}$ & Yes & - & +1 Month & Spine L4-L5 & $\begin{array}{l}\text { Plaster Jackets } \\
\text { including both } \\
\text { hips and thighs }\end{array}$ & 120 & - & 6 \\
\hline 57 & $10 / 10$ & $\mathrm{M}$ & $\mathrm{N}-\mathrm{W}$ & Yes & $\begin{array}{l}\text { Strong } \\
\text { Reactor }\end{array}$ & +2 Months & Spine L4-L5 & $\begin{array}{l}\text { Plaster Jackets } \\
\text { including both } \\
\text { hips and thighs }\end{array}$ & 180 & Iliopsoas Abscess Drainage & 71 \\
\hline 58 & $1 / 5$ & M. & $\mathrm{N}-\mathrm{W}$ & Yes & $\begin{array}{c}\text { No- } \\
\text { Reactor }\end{array}$ & +2 Months & Left Hip & Spica Cast & 90 & - & 7 \\
\hline 59 & $3 / 1$ & F. & W & Yes & $\begin{array}{c}\text { No- } \\
\text { Reactor }\end{array}$ & +1 Month & Spine L5-S1 & $\begin{array}{l}\text { Plaster Jackets } \\
\text { including both } \\
\text { hips and thighs }\end{array}$ & 100 & - & 6 \\
\hline 60 & $2 / 8$ & M. & $\mathrm{N}-\mathrm{W}$ & - & $\begin{array}{l}\text { Strong } \\
\text { Reactor }\end{array}$ & - 1 Month & $\begin{array}{l}\text { Spine T8- } \\
\text { T9-T10/Left } \\
\text { Elbow }\end{array}$ & $\begin{array}{c}\text { Thoracolumbar } \\
\text { Cast/ } \\
\text { Metacarpal } \\
\text { Heads to the } \\
\text { Axilla Cast }\end{array}$ & $\begin{array}{l}90 \\
30\end{array}$ & - & 6 \\
\hline 61 & $1 / 5$ & $\mathrm{~F}$ & $\mathrm{~N}-\mathrm{W}$ & Yes & - & - 1 Month & Spine L1-L2 & $\begin{array}{l}\text { Plaster Jackets } \\
\text { including both } \\
\text { hips and thighs }\end{array}$ & 90 & - & 6.3 \\
\hline 62 & $2 / 1$ & $\mathrm{~F}$ & $\mathrm{~N}-\mathrm{W}$ & Yes & $\begin{array}{l}\text { Strong } \\
\text { Reactor }\end{array}$ & +1 Month & Spine T9-T10 & $\begin{array}{l}\text { Plaster Jackets } \\
\text { including both } \\
\text { hips and thighs }\end{array}$ & 180 & - & 7.3 \\
\hline 63 & $1 / 11$ & M & $\mathrm{N}-\mathrm{W}$ & Yes & $\begin{array}{c}\text { No- } \\
\text { Reactor }\end{array}$ & +2 Months & Spine T12-L1 & $\begin{array}{l}\text { Plaster Jackets } \\
\text { including both } \\
\text { hips and thighs }\end{array}$ & 180 & - & 36.5 \\
\hline 64 & $3 / 6$ & M & $\mathrm{N}-\mathrm{W}$ & - & $\begin{array}{c}\text { No- } \\
\text { Reactor }\end{array}$ & - 1 Month & Spine T7-T8 & $\begin{array}{c}\text { Thoracolumbar } \\
\text { Cast }\end{array}$ & 105 & - & 18 \\
\hline
\end{tabular}

Citation: Carlos PP (2014) Spectrum of Skeletal Tuberculosis in Children. J Exerc Sports Orthop 1(2): 1-10. DOI: http://dx.doi. Page 7 of 10 org/10.15226/2374-6904/1/2/00109 


\begin{tabular}{|c|c|c|c|c|c|c|c|c|c|c|c|}
\hline 65 & $2 / 8$ & M & $\mathrm{N}-\mathrm{W}$ & - & \begin{tabular}{|l} 
Strong \\
Reactor
\end{tabular} & + 1 Month & Spine T5-T6 & $\begin{array}{c}\text { Thoracolumbar } \\
\text { Cast }\end{array}$ & 150 & - & 56.6 \\
\hline 66 & $2 / 0$ & M & $\mathrm{N}-\mathrm{W}$ & - & - & - 1 Month & Spine L3-L4 & $\begin{array}{c}\text { Thoracolumbar } \\
\text { Cast }\end{array}$ & 120 & - & 6 \\
\hline 67 & $1 / 7$ & $\mathrm{~F}$ & $\mathrm{~N}-\mathrm{W}$ & - & - & - 1 Month & Spine L5-S1 & $\begin{array}{l}\text { Plaster Jackets } \\
\text { including both } \\
\text { hips and thighs }\end{array}$ & 100 & - & 6.4 \\
\hline 68 & $5 / 5$ & $\mathrm{~F}$ & $\mathrm{~N}-\mathrm{W}$ & Yes & $\begin{array}{l}\text { Strong } \\
\text { Reator }\end{array}$ & + 3 Months & $\begin{array}{c}\text { Thotacolumbar } \\
\text { Spine }\end{array}$ & $\begin{array}{c}\text { Thoracolumbar } \\
\text { Cast }\end{array}$ & 120 & - & 97.3 \\
\hline 69 & $4 / 2$ & M. & $\mathrm{N}-\mathrm{w}$ & - & $\begin{array}{l}\text { Strong } \\
\text { Reactor }\end{array}$ & - 1 Month & Left Hip & Spica Cast & 30 & $\begin{array}{c}\text { Puncture/Artrotomy/ } \\
\text { Biopsy }\end{array}$ & 4.6 \\
\hline 70 & $2 / 0$ & $\mathrm{~F}$ & $\mathrm{~N}-\mathrm{W}$ & Yes & $\begin{array}{l}\text { Strong } \\
\text { Reactor }\end{array}$ & +1 Month & Spine T9-T10 & $\begin{array}{l}\text { Plaster Jackets } \\
\text { including both } \\
\text { hips and thighs }\end{array}$ & 120 & - & 12 \\
\hline 71 & $3 / 0$ & $\mathrm{~F}$ & $\mathrm{~N}-\mathrm{W}$ & - & $\begin{array}{c}\text { No- } \\
\text { Reactor }\end{array}$ & + 1 Month & Spine L2-L3 & $\begin{array}{l}\text { Plaster Jackets } \\
\text { including both } \\
\text { hips and thighs }\end{array}$ & $=100$ & - & 6 \\
\hline 72 & $1 / 0$ & M & $\mathrm{N}-\mathrm{W}$ & Yes & $\begin{array}{l}\text { Weak } \\
\text { Reactor }\end{array}$ & - 1 Month & Spine L4-L5 & $\begin{array}{l}\text { Plaster Jackets } \\
\text { including both } \\
\text { hips and thighs }\end{array}$ & 210 & - & 9 \\
\hline 73 & $0 / 6$ & $\mathrm{~F}$ & $\mathrm{~N}-\mathrm{W}$ & - & $\begin{array}{l}\text { Strong } \\
\text { Reator }\end{array}$ & +2 Months & Left Hip & Spica Cast & 120 & - & 6.6 \\
\hline 74 & $1 / 7$ & M & $\mathrm{N}-\mathrm{W}$ & Yes & $\begin{array}{c}\text { No- } \\
\text { Reactor }\end{array}$ & - 1 Month & Spine L2-L3 & $\begin{array}{l}\text { Plaster Jackets } \\
\text { including both } \\
\text { hips and thighs }\end{array}$ & 90 & - & 6.1 \\
\hline 75 & $3 / 6$ & M & $\mathrm{N}-\mathrm{W}$ & Yes & $\begin{array}{l}\text { Strong } \\
\text { Reactor }\end{array}$ & +3 Months & Spine L1-L2-L3 & $\begin{array}{c}\text { Thoracolumbar } \\
\text { Cast }\end{array}$ & 180 & Iliopsoas Abscess Drainage & 9 \\
\hline 76 & $5 / 8$ & $\mathrm{~F}$ & $\mathrm{~N}-\mathrm{W}$ & Yes & \begin{tabular}{|l} 
Strong \\
Reactor
\end{tabular} & +3 Months & Right Hip & Spica Cast & 90 & $\begin{array}{c}\text { Puncture/Artrotomy/ } \\
\text { Biopsy }\end{array}$ & 20 \\
\hline 77 & $1 / 7$ & M & $\mathrm{N}-\mathrm{W}$ & - & $\begin{array}{l}\text { Strong } \\
\text { Reactor }\end{array}$ & +1 Month & Spine L3-L4 & $\begin{array}{l}\text { Plaster Jackets } \\
\text { including both } \\
\text { hips and thighs }\end{array}$ & 180 & 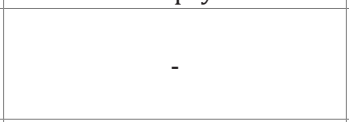 & 7.4 \\
\hline 78 & $3 / 5$ & $\mathrm{~F}$ & $\mathrm{~N}-\mathrm{W}$ & Yes & $\begin{array}{l}\text { Strong } \\
\text { Reactor }\end{array}$ & - 1 Month & Spine L1-L2 & $\begin{array}{l}\text { Plaster Jackets } \\
\text { including both } \\
\text { hips and thigh }\end{array}$ & 180 & & 9 \\
\hline 79 & $3 / 7$ & M & $\mathrm{N}-\mathrm{W}$ & - & - & +1 Month & Spine T5-T9 & $\begin{array}{l}\text { Plaster Jackets } \\
\text { including both } \\
\text { hips and thigh }\end{array}$ & 210 & - & 58 \\
\hline 80 & $1 / 7$ & M & $\mathrm{N}-\mathrm{W}$ & - & $\begin{array}{l}\text { Strong } \\
\text { Reactor }\end{array}$ & -1 Month & Right Femur & $\begin{array}{l}\text { Groin to foot } \\
\text { plaster cast }\end{array}$ & 90 & Puncture / Biopsy & 10 \\
\hline 81 & $5 / 11$ & $\mathrm{~F}$ & $\mathrm{~N}-\mathrm{W}$ & - & $\begin{array}{c}\text { No } \\
\text { Reactor }\end{array}$ & + 1 Month & Spine 11-L2 & $\begin{array}{l}\text { Plaster Jackets } \\
\text { including both } \\
\text { hips and thigh }\end{array}$ & 180 & - & 7 \\
\hline 82 & $8 / 0$ & M & W & - & $\begin{array}{l}\text { Strong } \\
\text { Reactor }\end{array}$ & +3 Months & $\begin{array}{l}\text { Left Wrist / } \\
\text { Dactylitis }\end{array}$ & - & - & - & 33 \\
\hline 83 & $6 / 11$ & $\mathrm{~F}$ & W & - & $\begin{array}{c}\text { No } \\
\text { Reactor }\end{array}$ & + 1 Month & Spine & $\begin{array}{l}\text { Plaster Jackets } \\
\text { including both } \\
\text { hips and thigh }\end{array}$ & 180 & - & 24.3 \\
\hline 84 & $5 / 8$ & $\mathrm{~F}$ & $\mathrm{~N}-\mathrm{W}$ & - & $\begin{array}{l}\text { Strong } \\
\text { Reactor }\end{array}$ & +3 Months & Right Hip & Spica Cast & 120 & - & 6.2 \\
\hline 85 & $2 / 9$ & $\mathrm{~F}$ & $\mathrm{~N}-\mathrm{W}$ & - & $\begin{array}{c}\text { No } \\
\text { Reactor }\end{array}$ & - 1 Month & Spine & $\begin{array}{l}\text { Plaster Jackets } \\
\text { including both } \\
\text { hips and thigh }\end{array}$ & 120 & - & 6.5 \\
\hline 86 & $1 / 9$ & $\mathrm{~F}$ & $\mathrm{~N}-\mathrm{W}$ & - & $\begin{array}{c}\text { No- } \\
\text { Reactor }\end{array}$ & + 1 Month & Spine L2-L3 & $\begin{array}{l}\text { Plaster Jackets } \\
\text { including both } \\
\text { hips and thigh }\end{array}$ & 120 & - & 22 \\
\hline 87 & $4 / 1$ & M. & $\mathrm{N}-\mathrm{W}$ & - & $\begin{array}{l}\text { Strong } \\
\text { Reactor }\end{array}$ & +2 Months & Spine T9-T10 & $\begin{array}{l}\text { Plaster Jackets } \\
\text { including both } \\
\text { hips and thigh }\end{array}$ & 180 & - & 40 \\
\hline
\end{tabular}

Citation: Carlos PP (2014) Spectrum of Skeletal Tuberculosis in Children. J Exerc Sports Orthop 1(2): 1-10. DOI: http://dx.doi. Page 8 of 10 org/10.15226/2374-6904/1/2/00109 


\begin{tabular}{|c|c|c|c|c|c|c|c|c|c|c|c|}
\hline 88 & $7 / 5$ & $\mathrm{~F}$ & $\mathrm{~N}-\mathrm{W}$ & - & $\begin{array}{l}\text { Strong } \\
\text { Reactor }\end{array}$ & +3 Months & Spine L4-L5 & $\begin{array}{l}\text { Plaster Jackets } \\
\text { including both } \\
\text { hips and thigh }\end{array}$ & 240 & - & 29 \\
\hline 89 & $4 / 0$ & $\mathrm{~F}$ & $\mathrm{~N}-\mathrm{W}$ & - & $\begin{array}{l}\text { Strong } \\
\text { Reactor }\end{array}$ & + 3 Months & Spine C2-C3 & $\begin{array}{l}\text { Minerva Jacket } \\
\text { Cervical Collar }\end{array}$ & $\begin{array}{c}40 \\
240\end{array}$ & - & 53.3 \\
\hline 90 & $1 / 4$ & $\mathrm{~F}$ & $\mathrm{~N}-\mathrm{W}$ & - & $\begin{array}{l}\text { Strong } \\
\text { Reactor }\end{array}$ & + 3 Months & Spine T10-T11 & $\begin{array}{l}\text { Plaster Jackets } \\
\text { including both } \\
\text { hips and thigh }\end{array}$ & 180 & - & 7 \\
\hline 91 & $2 / 9$ & M. & $\mathrm{N}-\mathrm{W}$ & - & $\begin{array}{l}\text { Strong } \\
\text { Reactor }\end{array}$ & + 1 Month & $\begin{array}{l}\text { Spine T9- } \\
\text { T10-T11 }\end{array}$ & $\begin{array}{l}\text { Plaster Jackets } \\
\text { including both } \\
\text { hips and thigh }\end{array}$ & 180 & $\begin{array}{l}\text { Drainage of iliopsoas } \\
\text { abscess muscle }\end{array}$ & 10 \\
\hline 92 & $1 / 4$ & M. & $\mathrm{N}-\mathrm{W}$ & - & $\begin{array}{l}\text { Strong } \\
\text { Reactor }\end{array}$ & +3 Months & Spine L3-L4 & $\begin{array}{l}\text { Plaster Jackets } \\
\text { including both } \\
\text { hips and thigh }\end{array}$ & 180 & Drainage of Lumbar abscess & 146.6 \\
\hline 93 & $2 / 11$ & M. & $\mathrm{N}-\mathrm{W}$ & - & $\begin{array}{l}\text { Strong } \\
\text { Reactor }\end{array}$ & +3 Months & Sternum & - & - & Puncture/biopsy & 10 \\
\hline 94 & $2 / 3$ & $\mathrm{~F}$ & W & - & - & +3 Months & Radius & $\begin{array}{l}\text { Metacarpal } \\
\text { Heads to the } \\
\text { Axilla Cast }\end{array}$ & 15 & Puncture/biopsy & 9 \\
\hline 95 & 10 & M & W & - & $\begin{array}{l}\text { Strong } \\
\text { Reactor }\end{array}$ & +3 Months & Neck & - & - & - & 24 \\
\hline 96 & 6 & M & W & - & $\begin{array}{l}\text { Strong } \\
\text { Reactor }\end{array}$ & +3 Months & Neck & - & - & - & 36 \\
\hline
\end{tabular}

to the tuberculin test used in patients bearing immunoreactivity characteristics [16].

In the literature, there is great expectation about the new and different forms of bone tuberculosis linked to AIDS. It is known that $29.5 \%$ of AIDS patients developed tuberculosis, with predominance of pulmonary tuberculosis [11]. In adults with AIDS living with children infected by HIV (human immunodeficiency virus), the increase of the same disease in adult population as well as in children is observed. Infection progression is faster in HIV-infected children by vertical transmission [17]. One patient infected by HIV was found in this study. However, conclusions cannot be taken as only nine patients presented negative specific serology.

The most frequent of osteoarticular tuberculosis is located in the spine. The percentage varies from $24 \%$ to $85 \%[1,6,14,18$ $20]$, followed by the hip, knee and ankle joints $[1,6,13,18]$. In this work, the spine, followed by the hip joint, according to the literature, were the most affected.

In relation to spondylitis tuberculosis, the most affected segments were the thoracic /lumbar spine, corresponding to (37\%), respectively. Other authors [14,19] also present a series of patients with a spondylitis tuberculosis diagnosis involving the lumbar spine with great frequency. Thoracic spine involvement is also quite prevalent, according to literature [20].

This consideration may be explained by pulmonary region proximity. In this work, such occurrence was found in twenty patients, which makes it unlikely to be spread by contiguity, as well as by hematogeneous and lymphatic via $[21,22]$.

Loembe et al. [14] found the occurrence of active pulmonary tuberculosis with bone in $25 \%$ of cases. In this study, association was observed in three cases (3.2\%). Taarit et al. [19] report that $27 \%$ of osteoarticular cases showed an involvement in two segments; in this study, such occurrence was observed in only three patients.

Conservative treatment should be employed for most patients with spinal tuberculosis. Exceptions for surgical treatment are reserved for certain situations, such as local infection spread, compression caused by tuberculosis abscesses, and involvement of three or more vertebral bodies, leading to mechanical instability. There was also marked kyphosis with progressive paraparesis/paraplegia, and persistent pulmonary and cardiac dysfunction $[1,3,20,23]$. Surgical procedure was indicated in those cases.

The Campos [15] principle considered it essential to improve the patient's clinical condition and then employ specific drugs combined with plaster cast application, thus favoring good disease prognostic. Furthermore, it stated that bed rest helped in the prevention of granulomatosous lesion spread [24,25].

Tuberculosis involvement of the sternum is rare in the literature, even in endemic countries. It accounts for less than $1 \%$ of musculoskeletal tuberculosis cases [26]. Diagnosis of a single case in this sample was made by puncture biopsy to confirm diagnosis.

The antituberculous drugs and cast immobilization in osteoarticular tuberculosis treatment bore satisfactory results. The applied method was simple and did not require prolonged hospitalization. An improvement of pain and neurological examination in a few days after initiating the multidrug chemotherapy treatment and cast regime immobilization for tuberculosis treatment were achieved. 


\section{Conclusions}

After analyzing the therapy method based on anti-tuberculous drugs and plaster cast immobilization, we concluded that employment of the standard protocol treatment, together with modern multidrug chemotherapy and cast were operational, justifiable and productive; it also can be used as an alternative therapeutic procedure regarding this specific disease.

\section{Acknowledgements}

The author wishes to thank Dr. João Ferreira Alves MD and Dr. Anna Machado Marques MD, for their assistance and help in preparing the manuscript.

\section{Author's information}

The author certifies that he has no commercial associations (e.g. consultancies, stock holdings, equity interest, patent/ licensing arrangements, etc) which pose a conflict of interest in connection with the submitted article.

\section{Competing Interests}

The author has not received any outside funding or grants in support for, or in preparation of his research. Neither did he, nor any member of his immediate family receive payments, benefits or agreements to provide the research for financial reasons.

\section{References}

1. Pinheiro PCMS, Bianco SM (1990) Tuberculose osteoarticular no Hospital Municipal Jesus nos últimos 17 anos. Rev Bras Ortop 25(5): 131-136.

2. Troncoso VME, Espinosa MLF, Ocampo LCB, Drusso LE (2000) Artritis tuberculosa. Rev Fac UNAM 43(6): 248-251.

3. Mandetta H, Pereira FA, Sismeiro LFF, Antoniolli R (1994) Mal de Pott : tratamento clínico e cirúrgico. Rev Bras Ortop 29(3): 139-143.

4. Sarinho ESC, Monte LA, Júnior OAFC (1991) Tuberculose osteoarticular no ambulatório de pediatria do H. C. - UFPE. Arq Bras Med 65(5): 472 474 .

5. Teklali Y, Alami ZF, Madhi T, Gourinda H, Miri A (2003) Peripheral osteoarticular tuberculosis in children: 106 case-reports. Joint Bone Spine70(4): 282-286.

6. Defino HLA, Oliveira W (1994) Problemas diagnósticos na tuberculose óssea: experiência da área de Ortopedia do HCFMRP-USP. Medicina( Ribeirão Preto)27(3/4): 345-351.

7. Rubin SA (1995) Tuberculosis: captain of these men of death. Radiol Clin North Am33(4): 619-639.

8. Pott P (1779) Remarks on that kind of palsy of the lower limbs which is frequently found to accompany a curvature of the spine. J Johnson, London.

9. Albee FH (1911) Transplantation of a portion of the tibia into the spine for Pott's disease. JAMA 57 (11): 885.

10. Hibbs RA (1911) An operation for progressive spinal deformities. NY Med J 93: 1013.

11. Pietrobon RS, Pinha MA, Costa PAB, Silva RF (1994) Epidemiologia da tuberculose óssea: análise de 149 casos no Paraná. Rev Bras Ortop29(6): 426-30.

12. Rasool MN (2001) Osseous manifestations of tuberculosis in children. J Pediatr Orthop21(6): 749-755.

13. Zamora CZ (1983) Tuberculosis osteoarticular en el Hospital Mexico.Acta med costarric26(2): 93- 99 .

14. Loembe PM, Assengone-Zeh Y, Guerch M, Mbumb-King A (1988) La tuberculose vertébrale au Gabon. Neurochirurgie 34(6): 420-427.

15. Campos OP (1955) Bone and joint tuberculosis and its treatment. J Bone Joint Surg Am37(5): 937-966.

16. Margolis ML, Van Uitert BL (1985) Anergy in tuberculosis. Biomed \& Pharmacotherapy39(6): 292-298.

17. Farhat CK, Carvalho ES (1998) Tuberculose. Infectologia pediátrica. Atheneu, Säo Paulo Brasil, pp. 343-52.

18. Jaime H, Patricio E, Dabor S, Carlos De la Barrera C, Rodolfo Nazar S, Jaime A (2001) Tuberculosis osteoarticular, experiência de 10 años. Rev Clin Univ Chile 12(1): 12-16.

19. Taarit CB, Maiz HB (2003) La tuberculose ostéoarticulaire en Tunisie: étude rétrospective de 180 cas. Médecine et maladies infectieuses 33(4): 210-2014.

20. Grosskopf I, David AB, Charach G, Hochman I, Pitlik S (1994) Bone and joint tuberculosis - A 10-year review. Isr J Med Sci 30(4): 278-283.

21. Fernandes SRM, Samara AM, Nardy SLM, Marques Neto JF (1984) Evolução clínica e conduta terapêutica na tuberculose osteoarticular. Rev Bras Reumat 24(2): 61-64.

22. Santos JF (2005) Tuberculosis in children. Europ J Radiol 55(2): 202-208.

23. Daher S, Cardoso ALP, Souza Jr ZA, Pimenta JRWE, Moraes FB, et al. (2006) Tuberculose espinhal: avaliação de 26 casos. Coluna 5(1): 1-6.

24. Griffith DL: Conservative treatment of tuberculosis of the spine: report of a medical research council working party. J Bone Joint Surg [Br] 1974. 56. pp. 197-198.

25. Pereira LF (1979) Tuberculose osteoarticular na criança [Thesis]. Faculdade de Medicina Universidade Federal do Rio de Janeiro, Rio de Janeiro.

26. Khan AS, Varshney MK, Hasan SA, Kumar A, Trikha V (2007) Tuberculosis of the sternum. J Bone Joint Surg (Br) 89(6): 817-820. 